\title{
A Hybrid Technique for Medical Image Segmentation
}

\author{
Alamgir Nyma, ${ }^{1}$ Myeongsu Kang, ${ }^{1}$ Yung-Keun Kwon, ${ }^{1}$ \\ Cheol-Hong Kim, ${ }^{2}$ and Jong-Myon Kim ${ }^{1}$ \\ ${ }^{1}$ School of Electrical Engineering, University of Ulsan, Building 7, Room No. 308, 93 Daehak-ro, Nam-gu, \\ Ulsan 680-749, Republic of Korea \\ ${ }^{2}$ School of Electronics and Computer Engineering, Chonnam National University, Building 7, Room No. 506, \\ 77 Yongbong-ro, Buk-gu, Gwangju 500-757, Republic of Korea
}

Correspondence should be addressed to Jong-Myon Kim, jmkim07@ulsan.ac.kr

Received 22 May 2012; Revised 11 July 2012; Accepted 12 July 2012

Academic Editor: Tai Hoon Kim

Copyright () 2012 Alamgir Nyma et al. This is an open access article distributed under the Creative Commons Attribution License, which permits unrestricted use, distribution, and reproduction in any medium, provided the original work is properly cited.

Medical image segmentation is an essential and challenging aspect in computer-aided diagnosis and also in pattern recognition research. This paper proposes a hybrid method for magnetic resonance (MR) image segmentation. We first remove impulsive noise inherent in MR images by utilizing a vector median filter. Subsequently, Otsu thresholding is used as an initial coarse segmentation method that finds the homogeneous regions of the input image. Finally, an enhanced suppressed fuzzy c-means is used to partition brain MR images into multiple segments, which employs an optimal suppression factor for the perfect clustering in the given data set. To evaluate the robustness of the proposed approach in noisy environment, we add different types of noise and different amount of noise to T1-weighted brain MR images. Experimental results show that the proposed algorithm outperforms other FCM based algorithms in terms of segmentation accuracy for both noise-free and noise-inserted MR images.

\section{Introduction}

Image segmentation plays a pivotal role in computer-aided diagnosis and therapy. The objective of image segmentation is to partition an image into nonoverlapping, constituent regions that are homogeneous with respect to some attributes such as intensity and texture [1]. The representative image segmentation methods are generally based on the following techniques: thresholding, clustering, and edge detection and region extraction [2]. In this study, a combination of both thresholding and fuzzy clustering techniques will be considered because thresholding finds out the uniform regions for fuzzy clustering that makes the clustering performance better.

For thresholding methods, it is necessary to convert an image to a binary image depending on threshold values that extract the objects from the background [3]. The most convenient and widely used technique is histogram thresholding that is based on the shape properties of the histogram. The image histogram has distinct peaks, with each peak corresponding to one distinct region, and the valleys as the threshold values for separating these regions $[4,5]$.
Thresholding-based segmentation algorithms are then generally efficient in terms of computational complexity when compared to other segmentation methods, and Otsu's clustering-based thresholding [6] is being one of the most representative methods for image segmentation.

In addition, fuzzy clustering has been widely studied and successfully applied in image clustering and segmentation [7]. Fuzzy c-means (FCM) clustering $[8,9]$, which is one of the basic methods of fuzzy clustering, is a soft segmentation method that has been used extensively to improve the compactness of the regions with its cluster validity and simple implementation. FCM depends on the Euclidean distance between pixels based on the assumption that each feature is of equal importance. However, in most real-world applications, features are not considered equally important. Thus, this assumption may seriously affect clustering performance. To improve the performance of FCM, many techniques have been proposed, such as rival checked FCM and suppressed FCM (SFCM), which integrates the hard c-means (HCM) and FCM in order to improve the convergence speed and clustering performance $[10,11]$. Based on the advantages of 
thresholding and fuzzy clustering algorithms for image segmentation, some authors have proposed hybrid techniques combining various FCM-based methods with thresholding. Histogram thresholding using fuzzy theory was proposed by Tobias and Seara [12] in which thresholding was done according to the similarity between grey levels, and this similarity was accessed through a fuzzy measure. However, the application of this algorithm is limited based on the assumption that objects and background must occupy nonoverlapping regions. Chaabane Ben et al. proposed a hybrid method that combines automatic thresholding with FCM [13]. However, this technique yielded good results such that significant peaks and valleys are identified properly. Another hybrid approach was introduced by Tan and Isa, and it provided a good solution to overcome the FCM's sensitiveness to the initialization condition of cluster centroids and selection of the number of clusters by using the histogram thresholding [14]. This algorithm, however, has one drawback such that some flat portions of the histogram curves had been recognized as dominating peaks. To improve the performance of medical image segmentation, we propose a hybrid technique using Otsu thresholding and enhanced SFCM (EnSFCM). Furthermore, we reduce impulsive noise that is widely presented in magnetic resonance (MR) images by employing vector median filtering.

The rest of this paper is organized as follows. Section 2 introduces the proposed image segmentation approach and Section 3 presents experimental results of the proposed approach and well-known image segmentation algorithms. Finally, Section 4 concludes this paper.

\section{Proposed Image Segmentation Framework}

The proposed image segmentation approach consists of vector median filtering, Otsu thresholding, and EnSFCM, as shown in Figure 1.

In the first step, vector median filtering is utilized to reduce impulsive noise in medical images. Otsu thresholding is then employed for rough segmentation of brain MR images, and finally EnSFCM is applied to have well-segmented images. More details about the proposed approach are described in the following sections.

2.1. Vector Median Filter. It is easily to see impulsive noise in medical images which is independent and uncorrelated to the image pixels and is also randomly distributed over the image. In the case of magnetic resonance (MR) images, uncertainty is widely presented because of impulsive noise in the partial volume effects originating from the low resolution of sensors [11, 15]. With these reasons, impulsive noise inherent in brain MR images can result in low segmentation performance. Thus this paper utilizes a vector median filter (VMF) to remove impulsive noise in images. VMF is a vector processing operator that has been introduced as an extension of scalar median filter and preserves the image without getting blurred and no shifting of boundary [16]. It approaches the problem of noise reduction by searching the most robust vector in the processing window. The process to find the most robust vector is as follows.
Denote by $I$ an image to be processed, and let $W$ be the processing window centered on the pixel under processing of size $N \times N, N=3,5,7, \ldots$, and so forth. Let us consider that each input vector $x_{i}$ is associated with the distance measure,

$$
L_{i}=\sum_{j=1}^{N}\left\|x_{i}-x_{j}\right\|_{\gamma} \quad \text { for } i=1,2, \ldots, N,
$$

where $\gamma$ represents the selected norm. The distance between two samples can be defined by

$$
\left\|x_{i}-x_{j}\right\|_{\gamma}=\left(\sum_{k=1}^{m}\left|x_{i k}-x_{j k}\right|^{\gamma}\right)^{1 / \gamma}
$$

where $m$ is the distance of the vectors and $x_{i k}$ is the $k$ th element of $x_{i}$. Thus distance $L_{i}$ serves as an ordering criterion of $L_{o} \leq L_{1} \leq \cdots \leq L_{N-1}$ which implies the same ordering of the input vectors. The VMF output of the set $\left\{x_{0}, x_{1}, \ldots, x_{N}\right\}$ is defined as the sample $x_{\mathrm{VMF}} \in\left\{x_{0}, x_{1}, \ldots, x_{N}\right\}$ that satisfies the following condition:

$$
\sum_{i=1}^{N}\left\|x_{\mathrm{VMF}}-x_{i}\right\|_{\gamma} \leq \sum_{i=1}^{N}\left\|x_{j}-x_{i}\right\|_{\gamma}, \quad \text { for } j=1,2, \ldots, N .
$$

2.2. Otsu Thresholding. The Otsu's algorithm is one of the most referenced thresholding methods to partition images by automatically selecting threshold values from the histogram of the image. To find threshold values, the Otsu's algorithm utilizes the variance property of the image because variance is the measure of uniformity; the greater value of variance represents the greater difference between the background and the object $[4,6]$. Initially, two regions are separated by the intensity threshold, and then the optimal threshold is determined by minimizing the within-class variance or maximizing the between-class variance.

Assume that $\left\{P_{i}\right\}_{i=0}^{L-1}$ are the probabilities of the graylevel image histogram of an image, where $L$ is the range of intensity levels. We can calculate the probabilities of background $\left(P_{B}\right)$ and the probabilities of object $\left(P_{O}\right)$ of the image with a threshold $t$ as follows:

$$
\begin{gathered}
P_{B}(t)=\sum_{i=0}^{t} P_{i}, \\
P_{O}(t)=1-P_{B}=\sum_{i=t+1}^{L-1} P_{i} .
\end{gathered}
$$

The mean associated with the background and the object can be further calculated using the following equations:

$$
\begin{gathered}
\mu_{B}(t)=\sum_{i=0}^{t} \frac{i \cdot P_{i}}{P_{B}(t)}, \\
\mu_{O}(t)=\sum_{i=t+1}^{L-1} \frac{i \cdot P_{i}}{P_{O}(t)} .
\end{gathered}
$$




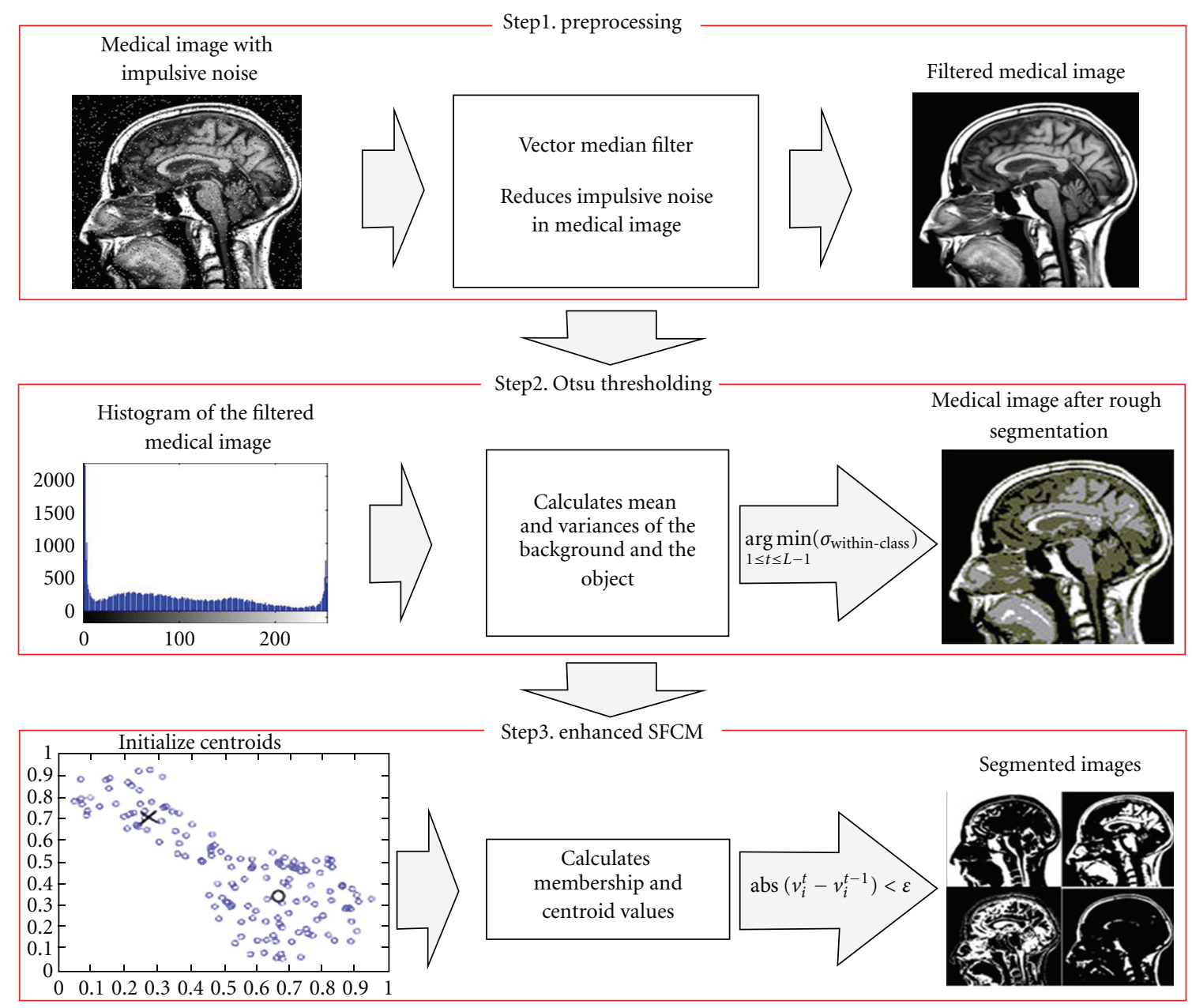

FIgURE 1: Process of the proposed image segmentation approach.

By using values of mean, we can compute variance as follows:

$$
\begin{gathered}
\sigma_{B}(t)=\sum_{i=0}^{t}\left(i-\mu_{B}\right)^{2} \cdot \frac{P_{i}}{P_{B}(t)}, \\
\sigma_{O}(t)=\sum_{i=t+1}^{L-1}\left(i-\mu_{O}\right)^{2} \cdot \frac{P_{i}}{P_{O}(t)} .
\end{gathered}
$$

The between-class variance $\left(\sigma_{\text {between-class }}(t)\right)$ which is the weighted variance of the cluster means around the overall mean is defined as follows:

$$
\sigma_{\text {between-class }}(t)=P_{B}\left(\mu_{B}(t)-\mu\right)^{2}+P_{O}\left(\mu_{O}(t)-\mu\right)^{2},
$$

where $\mu=\sum_{i=0}^{L-1} i \cdot P_{i}$ is the global mean of the image. Furthermore, the within-class variance $\left(\sigma_{\text {within-class }}(t)\right)$ can be expressed as follows:

$$
\sigma_{\text {within-class }}(t)=P_{B}(t) \times \sigma_{B}(t)+P_{O}(t) \times \sigma_{O}(t) .
$$

Finally, the optimal threshold value, $t_{\mathrm{opt}}$, can be determined by maximizing the between-class variance or equivalently minimizing the with-class variance as follows:

$$
\begin{aligned}
t_{\text {opt }} & =\underset{1 \leq i \leq L-1}{\arg \max }\left(\sigma_{\text {between-class }}(t)\right), \\
& =\underset{1 \leq i \leq L-1}{\arg \min }\left(\sigma_{\text {within-class }}(t)\right) .
\end{aligned}
$$

2.3. Enhanced Suppressed Fuzzy C-Means. Fuzzy c-means (FCM) is a data clustering algorithm in which data can belong to several groups based on the membership value, and it is one of the most prominent clustering techniques for image segmentation, feature extraction, and pattern recognition $[9,17]$. However, the conventional FCM sometimes leads to accuracy degradation in segmentation. To solve this problem, suppressed FCM (SFCM) was proposed to improve the clustering performance as well as convergence speed, which introduces the suppression factor for establishing a relationship between hard c-means (HCM) and FCM clustering algorithms [10]. However, there is no general consensus that the suppression factor for SFCM is optimal in any sense or suitable for any given purpose. Thus, we propose 
an enhanced suppressed FCM (EnSFCM) to automatically select the suppression factor for SFCM. The selection procedure of the suppression factor, $\alpha$, is based on the separation strength between clusters that is a time variant suppression rate. More details about EhSFCM are as follows.

Let $X=\left\{x_{1}, x_{2}, x_{3}, \ldots, x_{n}\right\}$ where $n$ is the number of image pixels. The conventional FCM algorithm sorts the data set $X$ into $c$ clusters. The standard FCM objective function is defined as follows:

$$
J_{m}(U, V)=\sum_{i=1}^{c} \sum_{k=1}^{n} \mu_{i k}^{m} d^{2}\left(x_{k}, v_{i}\right),
$$

where $d^{2}\left(x_{k}, v_{i}\right)$ is the Euclidian distance between the data point $x_{k}$ and the centroid $v_{i}$ of the $i$ th cluster and $\mu_{i k}$ is the degree of membership of the data $x_{k}$ to the $k$ th cluster. The parameter $m$ which is called the fuzzy factor controls the fuzziness of the resulting partition, $(m \geq 1)$, and $c$ is the total number of clusters. FCM clustering is an iterationbased clustering technique that produces an optimal number of $c$ classes by minimizing the objective function $J_{m}(U, V)$ with updated values of $\mu_{i k}$ and $v_{i}$ according to the following equations:

$$
\begin{gathered}
\mu_{i k}=\left[\sum_{j=1}^{c}\left(\frac{d^{2}\left(x_{k}, v_{i}\right)}{d^{2}\left(x_{k}, v_{j}\right)}\right)^{1 /(m-1)}\right]^{-1}, \\
v_{i}=\frac{\sum_{k=1}^{n} \mu_{i k}^{m} \cdot x_{k}}{\sum_{k=1}^{n} \mu_{i k}^{m}} .
\end{gathered}
$$

SFCM modifies the membership function in FCM by utilizing the suppression factor as follows:

$$
\begin{gathered}
\mu_{p k}=1-\alpha \sum_{i \neq p} \mu_{i k}=1-\alpha+\alpha \mu_{p k} \\
\mu_{p k}=\alpha \mu_{p k}, \quad i \neq p
\end{gathered}
$$

where $\mu_{p k}$ refers to data point, $x_{k}$ belongs to the largest cluster $p$, and $\alpha$ is the suppression factor which ranges in the interval $[0,1]$. SFCM then updates the value of $v_{i}$ with the new membership function. The pixel clustering iterations are terminated when the termination measurement $\max _{1 \leq i \leq c}\left\|v_{i}^{t}-v_{i}^{t-1}\right\|<\varepsilon$ is satisfied, where $v_{i}^{t}$ are the current centroids for $1 \leq i \leq c, v_{i}^{t-1}$ are the previous centroids for $1 \leq i \leq c$, and $\varepsilon$ is a predefined termination threshold. To match the perfect clustering of the given data set, it is necessary to select an optimal suppression factor for SFCM. We define a new exponential function to select the optimal suppression factor, and it is automatically updated at each iteration:

$$
\alpha=\exp \left(-\min _{i \neq j} \frac{\left\|v_{i}-v_{j}\right\|^{2}}{m}\right)
$$

where $v_{i}$ is the centroid of the $i$ th cluster, $v_{j}$ is the centroid of the $j$ th cluster, and $m$ is the degree of fuzzification. As mentioned before, selection of the suppression factor, $\alpha$, is very essential for SFCM since higher values of $\alpha$ indicate that FCM is superior to HCM whereas lower values represent vice versa. It is evident that good clustering performance exhibits compact clusters by giving more distance between centroids. Likewise, both the fuzzification parameter and the suppression rate $\alpha$ influence the learning rate of the algorithms. Thus we intuitively selected $\min _{i \neq j}\left\|v_{i}-v_{j}\right\|^{2} / m$, illustrating the separation strength between the clusters and the fuzzy factor that signifies the fuzziness of the membership values for the clustered data points.

\section{Experimental Results}

This section evaluates the performance of the proposed approach. To evaluate the performance of the proposed approach, we set some empirical parameters. Bezdek et al. experimentally determined the optimal intervals for the degree of fuzzification $(m)$ and the termination threshold $(\varepsilon)$, which ranged from 1.1 to 5 and 0.01 to 0.0001 , respectively [17]. In this paper, we selected $m=2$ and the termination threshold to be 0.0001 .

\subsection{Segmentation Results for Gray Matter and White Matter.} We evaluate the correctness of the segmentation using real brain scans with ground truth by expert segmentations obtained from the Internet Brain Segmentation Repository (IBSR) [18]. Figures 2(a)-2(b) and Figures 3(a)-3(b) show examples of two slices and the manual labeling provided by the IBSR, whereas the rest of the images illustrates the results of clustering by using FCM [9], MSFCM [11], FCMT [13], SFCM [19], and the proposed approach.

As indicated in the figures by a red mark, other FCMbased segmentation approaches miss considerable pixels in the red-marked region. On the other hand, the proposed approach successfully avoids this classification error. In addition to qualitative results, we quantitatively evaluate and calculate the segmentation accuracy of the proposed approach, which is described below.

3.2. Segmentation Accuracy. The performances of the proposed and other FCM-based approaches are compared with respect to the optimal segmentation accuracy [20], which is defined as the sum of the correctly classified pixels divided by the sum of the total number of pixels:

$$
\text { Segmentation Accuracy }=\sum_{i=1}^{c} \frac{A_{i} \cap C_{i}}{\sum_{j=1}^{c} C_{j}},
$$

where $c$ is the number of clusters, $A_{i}$ represents the set of pixels belonging to the $i$ th cluster by the segmentation algorithm, and $C_{i}$ represents the set of pixels belonging to the $i$ th cluster in the reference segmented image. To evaluate the robustness of the proposed segmentation approach in noisy environment, we add different types of noise and different amount of noise to a T1-weighted brain MR image, shown in Figure 2(a). All test images are corrupted by $8 \%$ and $10 \%$ Gaussian noise, speckle noise, and $10 \%$ and $12 \%$ salt and pepper noise as shown in Figure 4. Figure 4 also shows segmentation results of the proposed and other FCM-based approaches. 


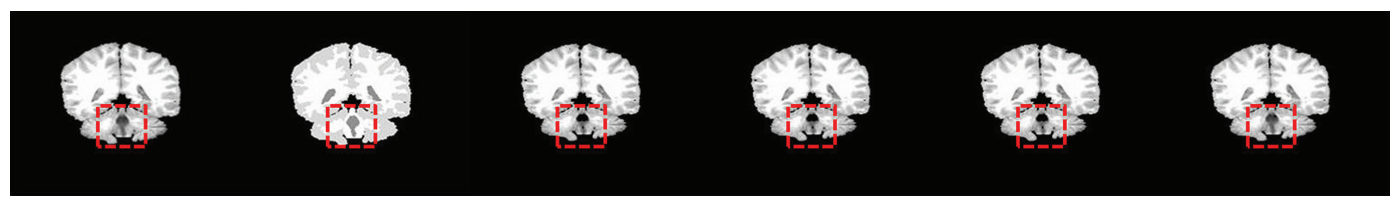

(a) (b)

(c)

(d)

(e)

(f)

FIgURE 2: Comparison of the segmentation results on a simulated brain MR image. (a) original T1-weighted image, (b) manual class labeling of gray matter (GM) and white matter (WM) slice regions; results obtained with (c) FCM, (d) MSFCM, (e) FCMT, (f) SFCM, and (g) the proposed approach.

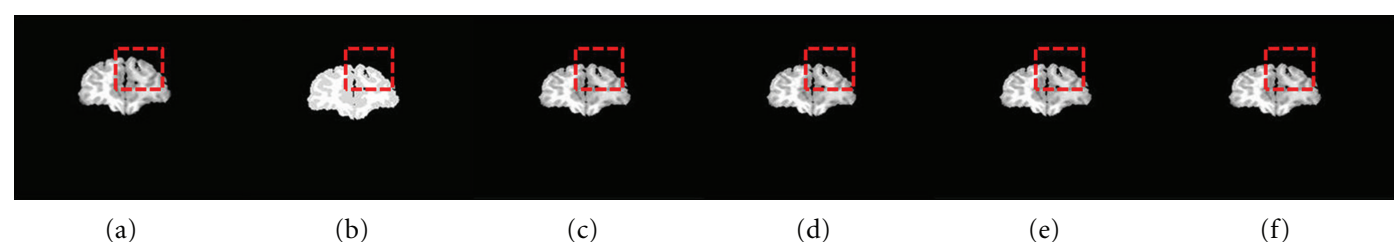

FIGURE 3: Comparison of the segmentation results on a simulated brain MR image. (a) original T1-weighted image, (b) manual class labeling of gray matter (GM) and white matter (WM) slice regions; results obtained with (c) FCM, (d) MSFCM, (e) FCMT, (f) SFCM, and (g) the proposed approach.

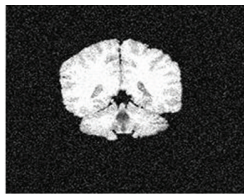

Original

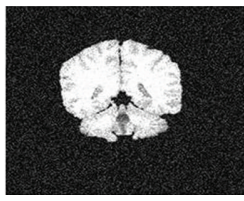

Original

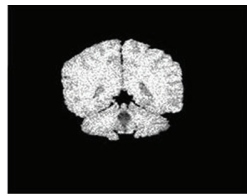

Original

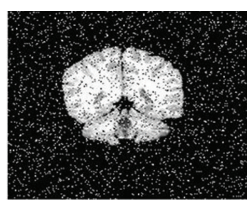

Original

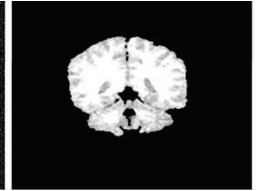

FCM

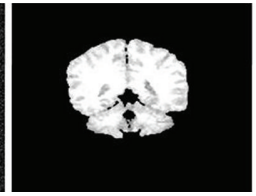

FCM

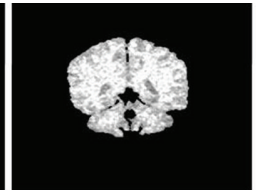

FCM

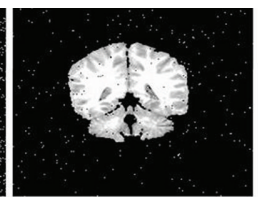

FCM

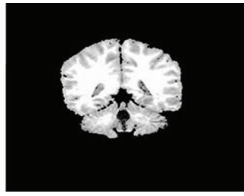

MSFCM

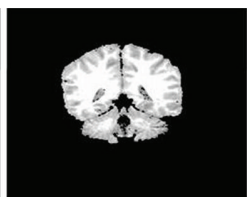

MSFCM

(b)

(a)

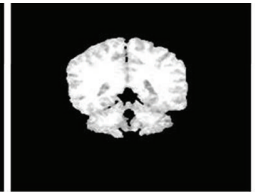

FCMT

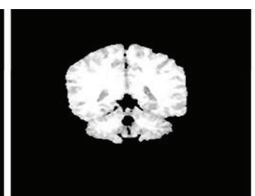

FCMT

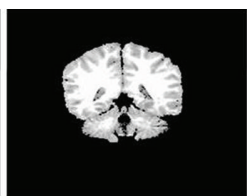

MSFCM

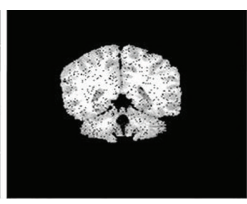

MSFCM

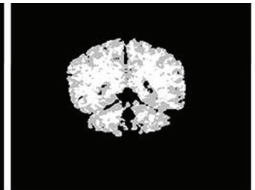

FCMT

(c)

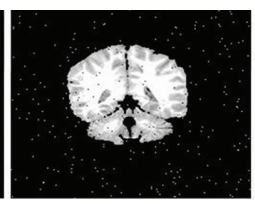

FCMT

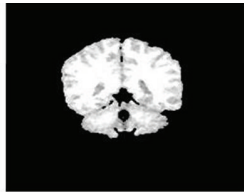

SFCM

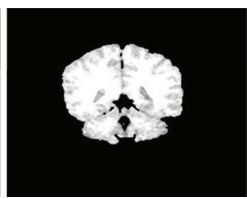

SFCM

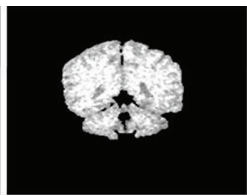

SFCM

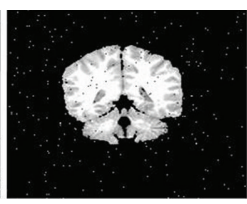

SFCM

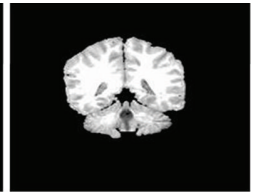

EnSFCM

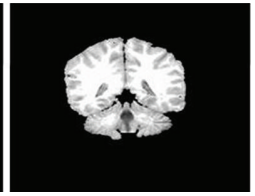

EnSFCM

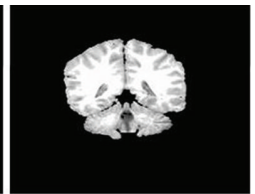

EnSFCM

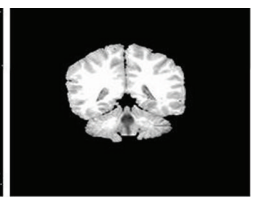

EnSFCM

(d)

FIGURE 4: Segmentation results in noisy environment. (a) 8\% Gaussian noise, (b) 10\% Gaussian noise, (c) speckle noise, (d) 10\% salt and pepper noise, and (e) $12 \%$ salt and pepper. 


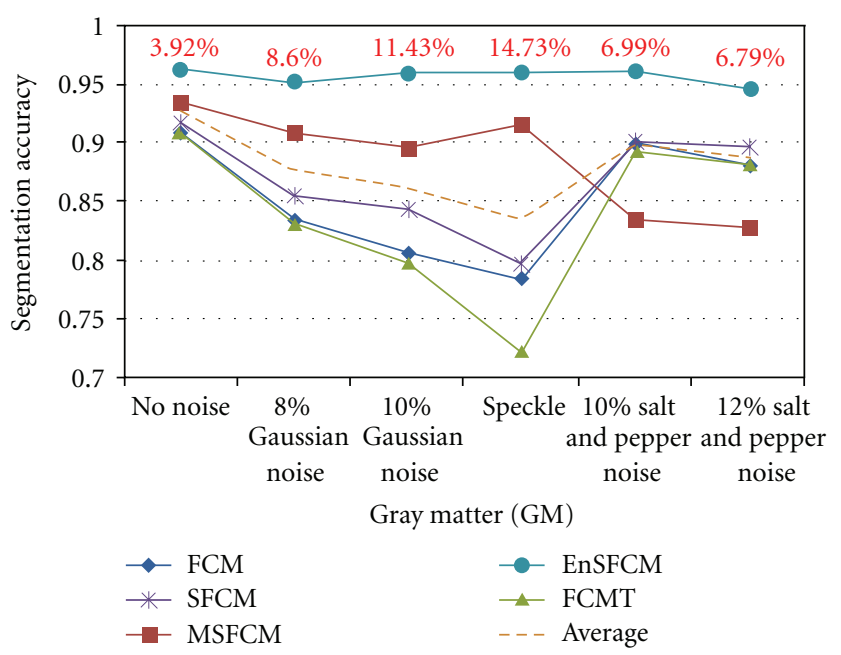

(a)

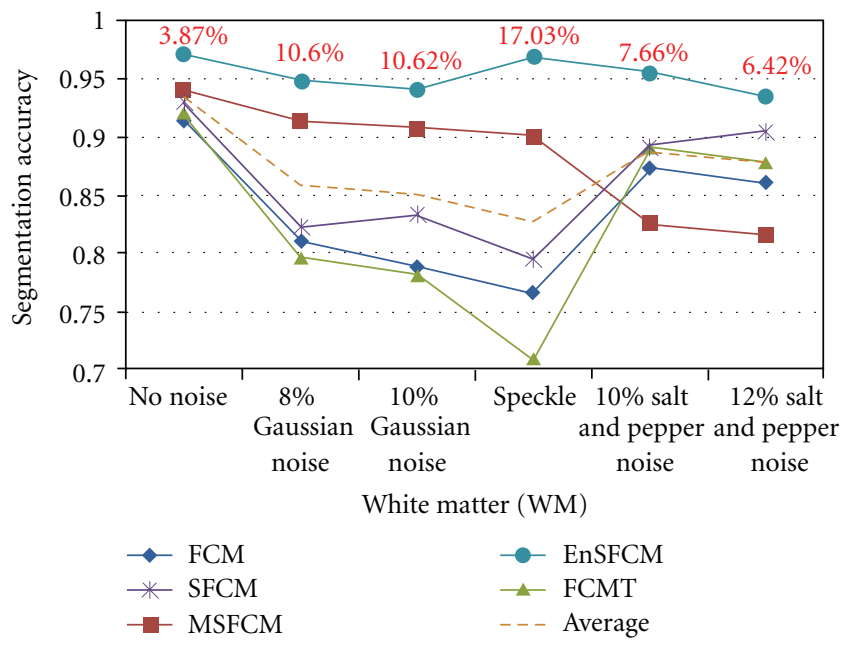

(b)

FIGURE 5: Segmentation accuracy results for gray matter (GM) and white matter (WM) in noisy environment by using FCM, MSFCM, FCMT, SFCM, and the proposed segmentation approach.

Figure 5 presents the quantitative results of these segmentation approaches for noise-free and noise-inserted brain MR images. The proposed algorithm increases 3.92\% (GM) and $3.87 \%$ (WM) of the segmentation accuracy for a noise-free brain MR image compared to the baseline performance, where we used the average value of the segmentation accuracy as a baseline. For noise-inserted images, the proposed algorithm achieves $6.79 \%-14.73 \%(\mathrm{GM})$ and $6.42 \%-$ $17.03 \%$ (WM) improvements in the segmentation accuracy, which is significant in the field of image segmentation for diagnosis purpose.

\section{Conclusions}

This paper presented a hybrid medical image segmentation technique. In the preprocessing step, we utilized vector median filtering to reduce the influence of noise. We then produced rough-segmented images using the Otsu thresholding method. To obtain well-segmented images, we finally used the enhanced suppressed FCM algorithm of the proposed approach. To match the perfect clustering of the given data set, we defined a new exponential function to automatically select the suppression factor for the suppressed FCM. To evaluate the robustness of the proposed segmentation approach in noisy environment, we added different types of noise and different amount of noise to the T1-weighted brain MR image and calculated the segmentation accuracy. Experimental results showed that the proposed method outperforms other FCM-based algorithms in terms of segmentation accuracy for both noise-free and noise-inserted images.

\section{Acknowledgments}

This work was supported by the National Research Foundation of Korea (NRF) grant funded by the Korean government (MEST) (no. 2012-000496, 2012-0001727).

\section{References}

[1] R. Gonzalez and R. Woods, Digital Image Processing, PrenticeHall, Upper Saddle River, NJ, USA, 2nd edition, 2002.

[2] K. S. Fu and J. K. Mui, "A survey on image segmentation," Pattern Recognition, vol. 13, no. 1, pp. 3-16, 1981.

[3] D. Jayadevappa, S. Srinivas Kumar, and D. S. Murthy, "A Hybrid segmentation model based on watershed and gradient vector flow for the detection of Brain tumor," International Journal of Signal Processing, Image Processing and Pattern Recognition, vol. 2, no. 3, pp. 29-42, 2009.

[4] H. S. Prasantha, H. L. Shashidhara, K. N. B. Murthy, and L. G. Madhavi, "Medical image segmentation," International Journal on Computer Science and Engineering, vol. 2, no. 4, pp. 1209-1218, 2010.

[5] Z. K. Huang, Y. M. Xie, D. H. Liu, and L. Y. Hou, "Using fuzzy c-means cluster for histogram-based color image segmentation," in Proceedings of the International Conference on Information Technology and Computer Science (ITCS '09), pp. 597600, July 2009.

[6] N. Otsu, "A Threshold selecting method from gray-level histograms," IEEE Transactions on Systems, Man, and Cybernetics, vol. 9, no. 1, pp. 62-66, 1979.

[7] M. Tabakov, "A fuzzy clustering technique for medical image segmentation," in Proceedings of the International Symposium on Evolving Fuzzy Systems (EFS '06), pp. 118-122, September 2006.

[8] J. C. Dunn, "A fuzzy relative of the ISODATA process and its use in detecting compact well-separated clusters," Journal of Cybernetics, vol. 3, no. 3, pp. 32-57, 1973.

[9] J. C. Bezdek, Pattern Recognition With Fuzzy Objective Function Algorithms, Advanced Applications in Pattern Recognition, Springer, Berlin, Germany, 1981.

[10] J. L. Fan, W. Z. Zhen, and W. X. Xie, "Suppressed fuzzy cmeans clustering algorithm," Pattern Recognition Letters, vol. 24, no. 9-10, pp. 1607-1612, 2003.

[11] W. L. Hung, M. S. Yang, and D. H. Chen, "Parameter selection for suppressed fuzzy c-means with an application to MRI segmentation," Pattern Recognition Letters, vol. 27, no. 5, pp. 424-438, 2006. 
[12] O. J. Tobias and R. Seara, "Image segmentation by histogram thresholding using fuzzy sets," IEEE Transactions on Image Processing, vol. 11, no. 12, pp. 1457-1465, 2002.

[13] S. Chaabane Ben, M. Sayadi, F. Fnaiech, and E. Brassart, "Color image segmentation using automatic thresholding and the fuzzy c-means techniques," in Proceedings of the IEEE Mediterranean Electrotechnical Conference (MELECON '08), pp. 857-861, May 2008.

[14] K. S. Tan and N. A. M. Isa, "Color image segmentation using histogram thresholding Fuzzy C-means hybrid approach," Pattern Recognition, vol. 44, no. 1, pp. 1-15, 2011.

[15] M. H. A. Hadi, K. Mortexza, and S. Y. Hadi, "Least mean square algorithm tuned by fuzzy C-means for impulsive noise suppression of gray-level images," International Journal of Signal Processing, Image Processing and Pattern Recognition, vol. 3, no. 4, pp. 55-66, 2010.

[16] J. Astola, P. Haavisto, and Y. Neuvo, "Vector median filters," Proceedings of the IEEE, vol. 78, no. 4, pp. 678-689, 1990.

[17] J. C. Bezdek, J. Keller, R. Krisnapuram, and N. Pal, Fuzzy Models and Algorithms For Pattern Recognition and Image Processing, Springer, Berlin, Germany, 2005.

[18] Internet Brain Segmentation Repository (IBSR).

[19] M. A. Ali, G. C. Karmakar, and L. S. Dooley, "Image segmentation using fuzzy clustering incorporating spatial information," in Proceedings of the International Conference on Computer and Information Technology, pp. 878-881, February 2004.

[20] L. Chen, C. L. P. Chen, and M. Lu, "A multiple-Kernel Fuzzy Cmeans algorithm for image segmentation," IEEE Transactions on Systems, Man, and Cybernetics, Part B, vol. 41, no. 5, pp. 1263-1274, 2011. 

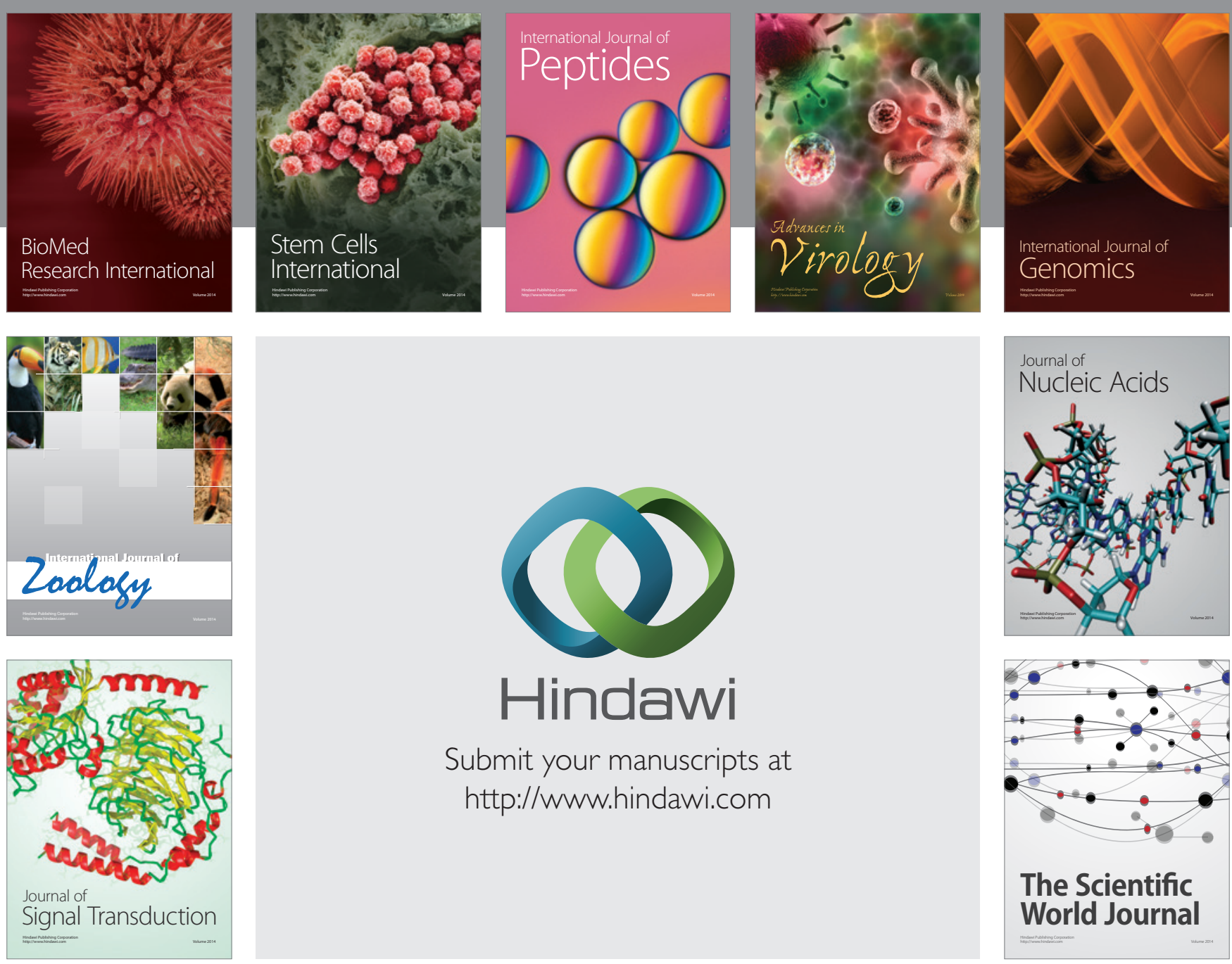

Submit your manuscripts at

http://www.hindawi.com
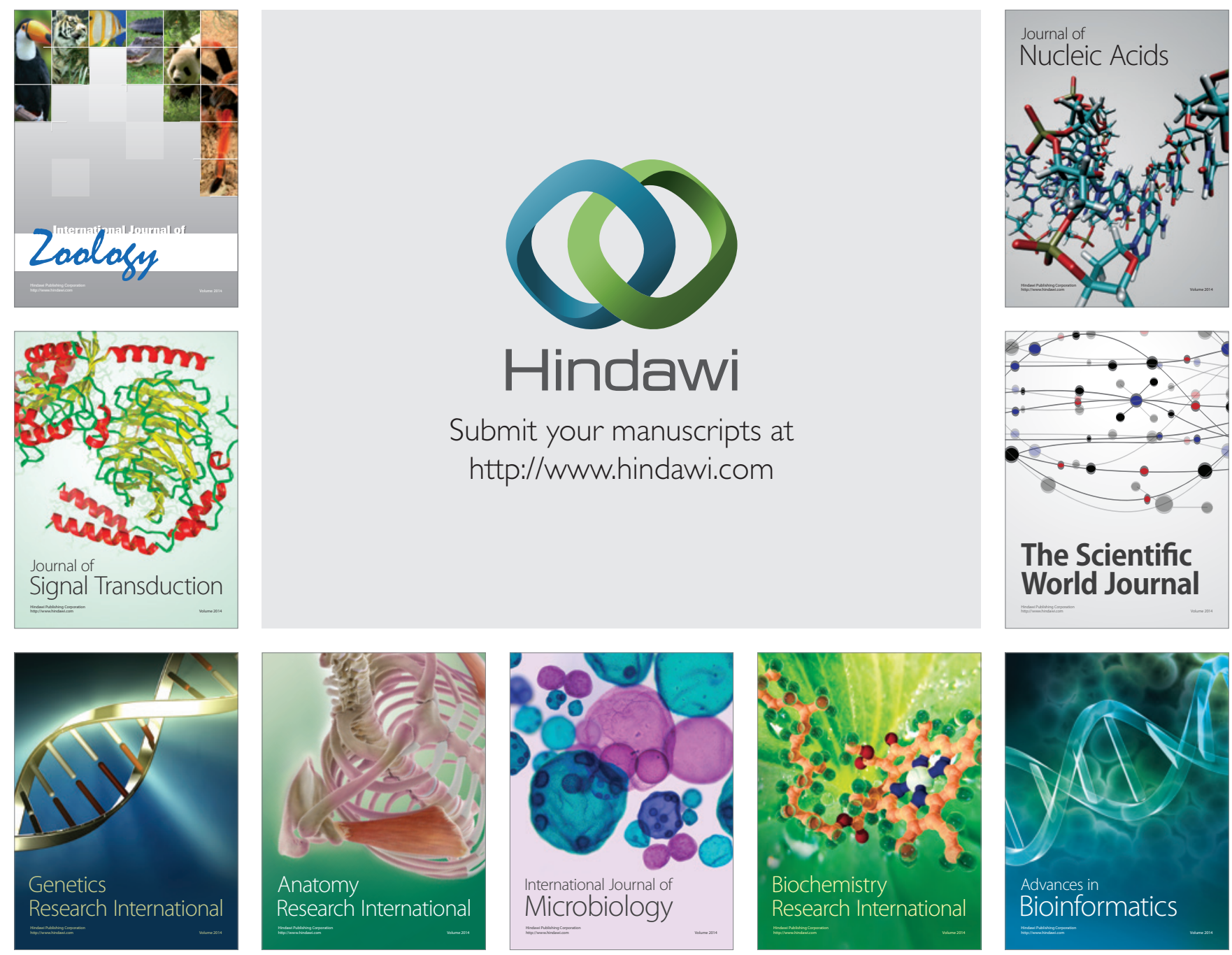

The Scientific World Journal
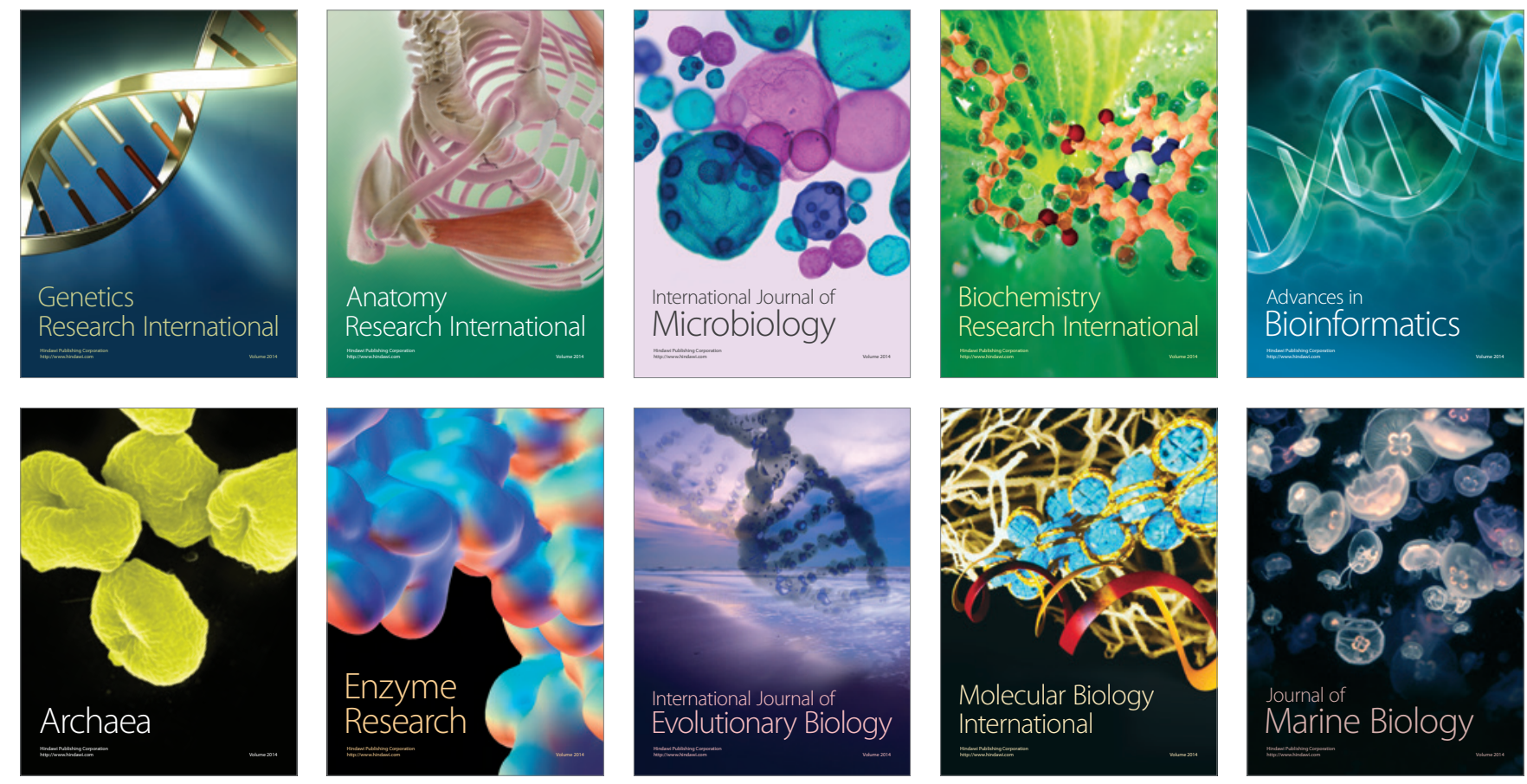